\title{
Efecto de extractos de Chenopodium album L. sobre los estados larval y adulto de Oryzaephilus surinamensis L. (Coleoptera: Silvanidae)
}

\author{
Effect of extracts of Chenopodium album L. on the larval and adult states of \\ Oryzaephilus surinamensis L. (Coleoptera: Silvanidae)
}

\author{
Serafina Russo ${ }^{1}$; Margarita Yaber Grass ${ }^{2}$; Silvia R. Leicach ${ }^{2}$
}

\begin{abstract}
RESUMEN
Para evaluar el efecto biológico de los metabolitos secundarios polares y no polares de la parte aérea (hojas e inflorescencias) de Chenopodium album L. (quinoa blanca) en la alimentación de Oryzaephilus surinamensis L. (Coleoptera: Silvanidae) se desarrolló un ensayo donde material vegetal previamente secado y triturado se sometió a extracción continua primero con éter de petróleo y luego con metanol, finalmente se realizó una extracción con agua en "batch" del material remanente dando origen a los correspondientes extractos. Se impregnaron $2 \mathrm{~g}$ de dieta base con solución de los extractos etéreo, metanólico y acuoso, en una concentración de $66 \mathrm{mg} / \mathrm{ml}$. En los tratamientos control se utilizó éter de petróleo metanol y agua, respectivamente. Se realizaron seis tratamientos en un diseño completamente aleatorizado, con cuatro repeticiones, analizándose el comportamiento y evolución de los insectos y el ciclo biológico de las larvas resultantes de los mismos. Cada siete días se determinó el número de individuos adultos y larvas vivas. Todos los datos fueron evaluados por ANOVA y las diferencias entre medias fueron verificadas por el test de Tukey $(\mathrm{p}<0,05)$. Los resultados indican que el extracto acuoso de C. album demostró ser el más efectivo con menor porcentaje de supervivencia de individuos adultos y particularmente de larvas con respecto a los demás tratamientos.
\end{abstract}

Palabras clave: Chenopodium album, Oryzaephilus surinamensis, metabolitos secundarios, extracto acuoso.

\begin{abstract}
The present assay was performed in order to evaluate the biological effects of polar and non-polar metabolites form the aerial parts of (leaves and inflorescences) Chenopodium album L. (white quinoa) on Oryzaephilus surinamensis L (Coleoptera: Silvanidae) feeding. Previously dried and ground plant material was submitted to sequential continuous extraction with petroleum ether and then with methanol; the remaining plant material was finally extracted in batch with water, giving rise to the corresponding extracts. $2 \mathrm{~g}$ of basic diet were impregnated with $66 \mathrm{mg} / \mathrm{ml}$ solutions of ether, methanol or aqueous extracts. Petroleum ether, methanol and distilled water were used respectively as control treatments. Six treatments were performed in a completely randomized design with four repetitions, analyzing insect behavior and the evolution of the biological cycle of the resulting larvae. The number of living adults and larvae was determined every seven days. All treatments were evaluated by means of ANOVA, and differences between mean values using Tukey`s test. $(p<0.05)$. Our results demonstrate that the aqueous extract of $\mathrm{C}$. album was the most effective, resulting in the lowest survival, particularly of larvae.
\end{abstract}

Key words: Chenopodium album, Oryzaephilus surinamensis, secondary metabolites, aqueous extract.

\section{Introducción}

Oryzaephilus surinamensis (Linnaeus, 1758) (Coleoptera: Silvanidae) es uno de los insectos más comunes en granos y productos almacenados. Está presente en regiones tropicales y subtropicales; tolerando bajas temperaturas en los climas templados. El adulto presenta cuerpo alargado, plano, de color castaño oscuro, midiendo 2,5 a $3,5 \mathrm{~mm}$ de longitud. En el tórax presenta seis dientes claramente

1 Facultad de Agronomía de la Universidad de Buenos Aires, Departamento de Producción Vegetal. Cátedra de Zoología Agrícola. Ciudad Autónoma de Buenos Aires, Argentina. Correo electrónico: srusso@agro.uba.ar

2 Facultad de Agronomía de la Universidad de Buenos Aires, Departamento de Biología Aplicada y Alimentos. Cátedra de Química de Biomoléculas. Ciudad Autónoma de Buenos Aires, Argentina. Correo electrónico: yaber@agro.uba.ar, leicach@ agro.uba.ar 
visibles en los bordes laterales y tres protuberancias longitudinales en forma de lomo en la parte central (Saini y Rodríguez, 2000). Es una plaga de infestación secundaria en granos debido a que es incapaz de dañar al grano entero, aunque los daños mecánicos producidos durante el almacenaje y secado producen la ruptura de granos, facilitando la entrada de estos insectos (Pricket et al., 1990). Tanto las larvas como los adultos son capaces de dañar productos almacenados con muy bajo contenido de humedad, pudiendo alimentarse de granos de cereales como trigo, maíz, arroz, cebada, sorgo y sus derivados, frutas secas, higos, uvas, cacao, nueces, alimentos para ganado (Mowery et al., 2002). Es un insecto muy pequeño que ha aumentando su resistencia a los insecticidas, y tiene la habilidad de ocultarse en diversos lugares en las instalaciones de almacenaje, haciendo difícil la entrada de los fitoterápicos (Wallbank and Collins, 2003).

El resurgimiento de las investigaciones sobre las posibilidades de insecticidas botánicos se debe al desarrollo de resistencia a los productos sintéticos en insectos plaga, uno de los problemas que han aparecido debido al uso indiscriminado de insecticidas sintéticos, además de otros como la contaminación ambiental y de alimentos y los efectos perjudiciales sobre los organismos benéficos.

Especies vegetales pertenecientes a las familias de Meliaceae, Labiatae, Umbeliferae, Compositae, entre otras, se destacan como promisorias para el desarrollo de nuevos insecticidas de origen vegetal (Olivera, 1997; Jacobson, 1989; Miana et al., 1996; Silva Aguayo, 2001). La familia Chenopodiaceae comprende alrededor de 100 géneros y 1.500 especies (Tousarkissian, 1980), que se caracterizan por contener diferentes grupos de metabolitos secundarios de los cuales los más importantes son los alcaloides, esteroides, flavonoides, fenoles y saponinas (Ibrahim, 2007). Estas sustancias, que están distribuidas en diferentes órganos de la planta, principalmente en raíces, hojas y semillas, pueden interferir en el metabolismo de otros organismos, causando efectos variables, como fagorrepelencia, esterilización, bloqueo del metabolismo e interferencia en la metamorfosis sin necesariamente causar la muerte (Lancher, 2000). Se ha confirmado la presencia de antocianas e isoflavonas en especies de las familias Caprifoliaceae, Labiatae, Verbenaceae y Chenopodiaceae (Woldu y Abegaz, 1990; Wollenweber, 1994; Donnelly y Boland, 1994).
Las diferentes especies del género Chenopodium poseen alternativamente propiedades terapéuticas y/o comestibles. Algunos autores mencionan el uso de partes aéreas de plantas de este género en la medicina folklórica, tal es el caso de $C$. graveolens utilizado en el tratamiento de algunas enfermedades producidas por microorganismos (Hernández et al., 2000). Investigaciones previas han demostrado que las saponinas de la quinoa exhiben actividad antifúngica y bactericida (Dutcheshen, 2004; Enviromental Protection Agency, 2005). Las propiedades insecticidas de aceites esenciales de $C$. ambrosioides se confirmaron sobre algunas especies de importancia en invernáculos: Myzus persicae, Frankliniella occidentales, Trialeurodes vaporariorium (Chiasson et al., 2004). Tradicionalmente $C$. album ha sido utilizado desde centurias como diurético, laxante, sedante, hepatoprotector y por sus propiedades antiparasitarias (Said, 1969; Fournier, 1999).

C. album, "quinoa blanca" es una planta anual, erecta de hasta $2 \mathrm{~m}$ de altura que posee hojas alternas irregularmente dentadas. Se encuentra distribuida en Argentina en una vasta zona que comprende desde La Rioja hasta Santa Cruz, siendo maleza de cultivos extensivos como trigo, arroz, girasol y lino (Marzocca, 1997).

La actividad antihelmíntica de $C$. album se comprobó tanto in vitro como in vivo, justificando su uso en medicina popular (Jabbar, 2007). Sus efectos inhibitorios sobre la germinación y el desarrollo de plántulas de soja fue también evaluado (Leicach y col., 2006), lo mismo que su actividad insecticida. Se examinó la toxicidad por contacto sobre Leptinotarsa decemlineata, de extractos de 30 plantas, entre las que figura $C$. album habiéndose comprobado que esta especie presentaba la máxima toxicidad, matando el 34,9\% de larvas después de las $72 \mathrm{~h}$ de aplicado el producto (Gökce et al., 2007). Otros estudios fueron realizados para determinar el efecto de distintas concentraciones de mezclas de material vegetal molido (inflorescencias, hojas y mezcla de ambas) de $C$. album adicionadas a la dieta base de Oryzaephilus surinamensis, encontrándose que las hojas poseían mayor impacto sobre los individuos adultos, mientras que las inflorescencias afectaban en mayor proporción a las formas juveniles de la plaga (Rodríguez et al., 2004). El ensayo se realizó con el fin de determinar algunas características estructurales de los compuestos bioactivos a partir de su solubilidad en solventes de polaridad creciente. Se analizó el efecto biológico de tres extractos de 
polaridad creciente obtenidos secuencialmente de hojas e inflorescencias de Chenopodium album L. (quinoa blanca) sobre la alimentación de Oryzaephilus surinamensis L. (Coleoptera: silvanidae).

\section{Materiales y Métodos}

El ensayo se desarrolló en laboratorios de las Cátedras de Química de Biomoléculas y Zoología Agrícola de la Facultad de Agronomía de la Universidad de Buenos Aires. Se trabajó con hojas e inflorescencias de plantas de C. album, que fueron recolectadas en el predio de la Facultad de Agronomía. El material vegetal se extrajo utilizando un equipo Soxhlet, previamente fue secado en estufa a $40{ }^{\circ} \mathrm{C}$ durante $48 \mathrm{~h}$ y luego molido. Se emplearon solventes de polaridad creciente, primero éter de petróleo (que retuvo los componentes menos polares) y luego metanol. Finalmente se realizó una extracción en batch con agua destilada. A partir de los extractos obtenidos, se prepararon soluciones en los correspondientes solventes en una concentración de $66 \mathrm{mg} / \mathrm{ml}$.

Para la cría y multiplicación de los insectos de $O$. surinamensis se emplearon recipientes de vidrio de boca ancha de $3 \mathrm{~cm}$ de diámetro y $4 \mathrm{~cm}$ de altura, con tapa de malla, ofreciendo una dieta constituida por harina de trigo tipo 0000, levadura seca de cerveza (Virgen, CALSA) y fécula de maíz (Maicena), en proporciones 10:1, 5:10 (Casadío y Zerba, 1996). Los insectos se mantuvieron en condiciones controladas de temperatura $\left(28{ }^{\circ} \mathrm{C}\right.$ $\left.\pm 3{ }^{\circ} \mathrm{C}\right)$ y humedad relativa $(75 \% \pm 5 \%)($ Sing y Moore, 1985).

La unidad experimental fue un recipiente de vidrio de $14 \mathrm{~cm}$ de diámetro por $5 \mathrm{~cm}$ de altura conteniendo dieta base ( $2 \mathrm{~g})$ a la que se adicionó el extracto a probar. En todos los casos en que se utilizaron solventes orgánicos, los recipientes se dejaron abiertos durante la noche, con el fin de permitir la total eliminación de los mismos por evaporación. Se colocaron 10 (diez) ejemplares adultos de carcoma dentada en cada uno de los frascos conteniendo el alimento al que se adicionó 1 $\mathrm{ml}$ de cada uno de los extractos (metanólico, etéreo y acuoso). Para la obtención de la descendencia se colocaron los adultos sobrevivientes de cada repetición (dentro del mismo tratamiento) en frascos de vidrio de $6 \mathrm{~cm}$ de ancho y $10 \mathrm{~cm}$ de altura conteniendo $10 \mathrm{~g}$ de dieta base. Se mantuvieron en condiciones controladas de temperatura $\left(25 \pm 3^{\circ} \mathrm{C}\right)$, humedad. $(75 \pm 5 \%)$ y en oscuridad durante 10 días hasta la aparición de las larvas neonatas. Las mismas se colocaron en número de diez en frascos de vidrio de $3 \mathrm{~cm}$ de ancho y $4 \mathrm{~cm}$ de altura conteniendo $2 \mathrm{~g}$ de dieta base.

Se utilizó un diseño completamente aleatorizado con cuatro repeticiones, para los siguientes tratamientos:

T1: Dieta base + Extracto etéreo de quinoa.

T2: Dieta base + Extracto metanólico de quinoa.

T3: Dieta base + Extracto acuoso de quinoa.

T4: Dieta base + éter de petróleo (Testigo éter).

T5: Dieta base + metanol (Testigo metanol).

T6: Dieta base + agua (Testigo agua).

Cada 7 días se evaluó periódicamente el número de adultos y larvas sobrevivientes durante 70 días. Con estos datos se calculó la supervivencia (\%) medida sobre el total de individuos colocados en cada uno de los ensayos al comienzo de los tratamientos.

$\%$ supervivencia $=\begin{gathered}\mathrm{N}^{\circ} \text { de individuos vivos } \times 100 / \mathrm{N}^{\circ} \\ \text { inicial individuos }\end{gathered}$

Los diferentes tratamientos fueron evaluados por ANOVA. Las diferencias entre medias fueron verificadas por el test de Tukey $(\mathrm{p}<0,05)$.

\section{Resultados}

$\mathrm{Al}$ analizar el porcentaje de supervivencia de los individuos adultos en función del tiempo se puede observar que el tratamiento T3 (extracto acuoso de quinoa) se diferencia significativamente de todos los demás (Figura 1) hasta los primeros 42 días de haber comenzado el ensayo; a partir de esa observación los tratamientos T3 (extracto acuoso de quínoa) y T2 (extracto metanólico de quínoa) no se diferenciaron entre sí, pero sí lo hacen con el T1 (extracto etéreo de quínoa) y con los testigos (Figura 1).

Al comparar los tratamientos se advierte claramente que el tratamiento T3 (extracto acuoso) es el que produce menor supervivencia (33\%) a los setenta días, mientras que para el tratamiento T2 (extracto metánolico) dicho parámetro es algo mayor (35\%), siendo el tratamiento T1 (extracto etéreo) el que permitió un mayor valor $(66,66 \%)$ de supervivencia (Figura 2). 
$\mathrm{Al}$ examinar la supervivencia de las formas juveniles (larvas) en función del tiempo se advierte nuevamente que T3 (extracto acuoso) presenta mayor eficacia con respecto a los otros tratamientos y los testigos. En este caso se puede observar una supervivencia menor en la totalidad de las observaciones realizadas (Figura 3). Al comparar todos los tratamientos se verifica nuevamente la mayor eficacia para T3 con una supervivencia del 5,19\%, mientras que el T1 (extracto etéreo de quínoa) y el T2 (extracto metánolico de quínoa) presentan supervivencia de 40,7 y 28,11, respectivamente (Figura 4).

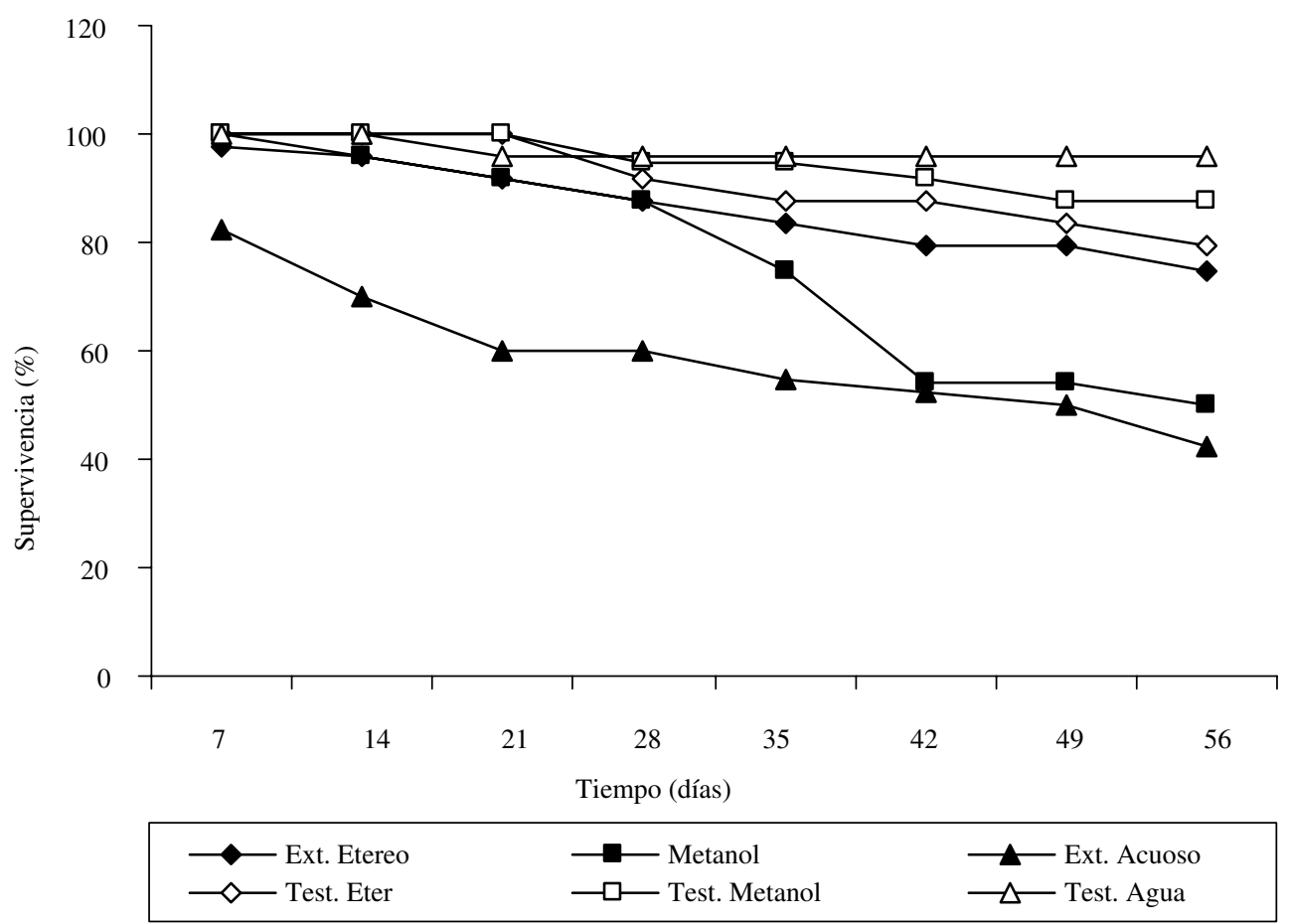

Figura 1. Supervivencia (\%) de individuos adultos en función del tiempo.

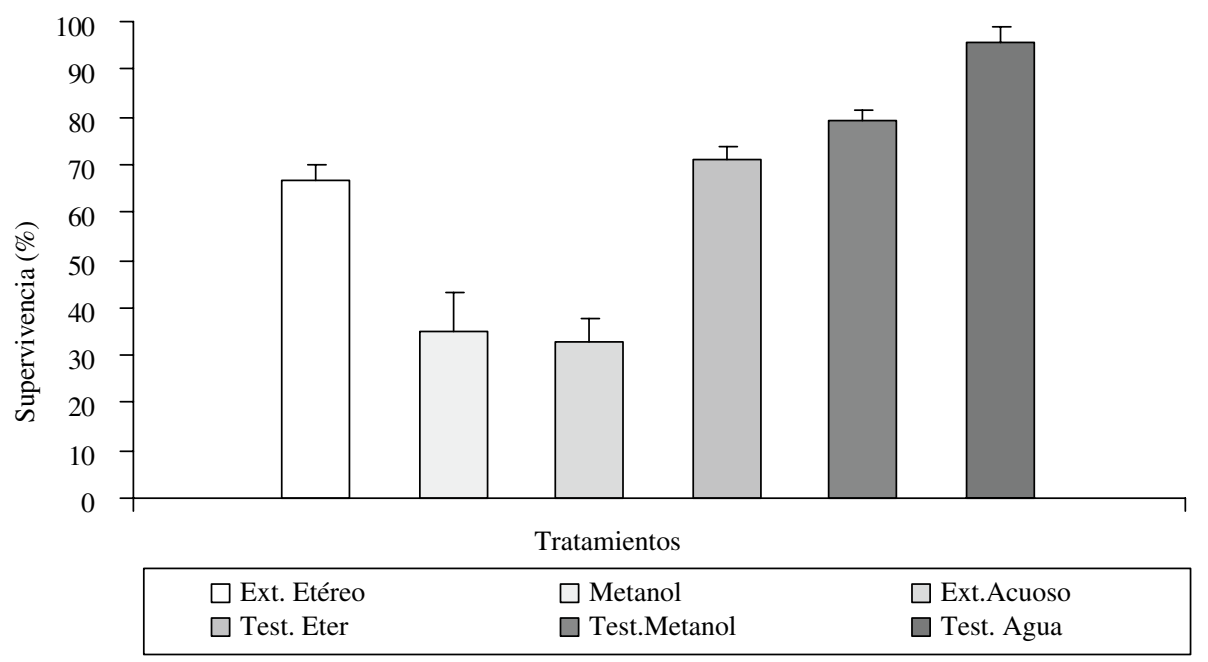

Figura 2. Supervivencia (\%) de individuos adultos de $O$. surinamensis. 
Llama la atención la muerte de una cantidad significativa de larvas en el control agua a partir de los 50 días, que podría deberse a algún cambio no advertido en el medio en que se realizó el ensayo. Cabe mencionar que las larvas son en general más susceptibles que los individuos adultos a ciertas modificaciones del entorno.

\section{Conclusiones}

Al comparar los resultados con los obtenidos por Rodríguez y colaboradores (2004), se verifica que la extracción secuencial permitió caracterizar a los compuestos más bioactivos dentro de los extractos más polares, debido al aumento en la concentración

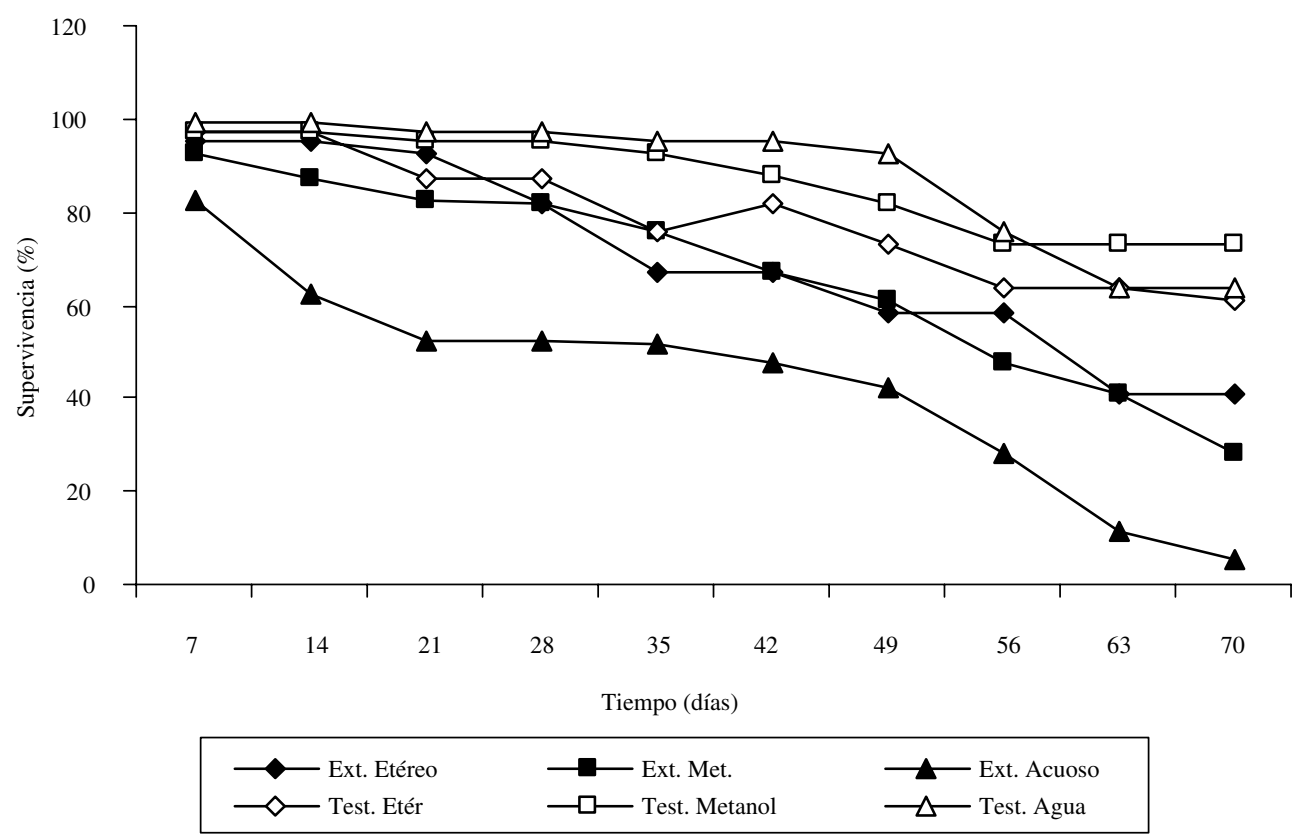

Figura 3. "Supervivencia (\%) de larvas en función del tiempo".

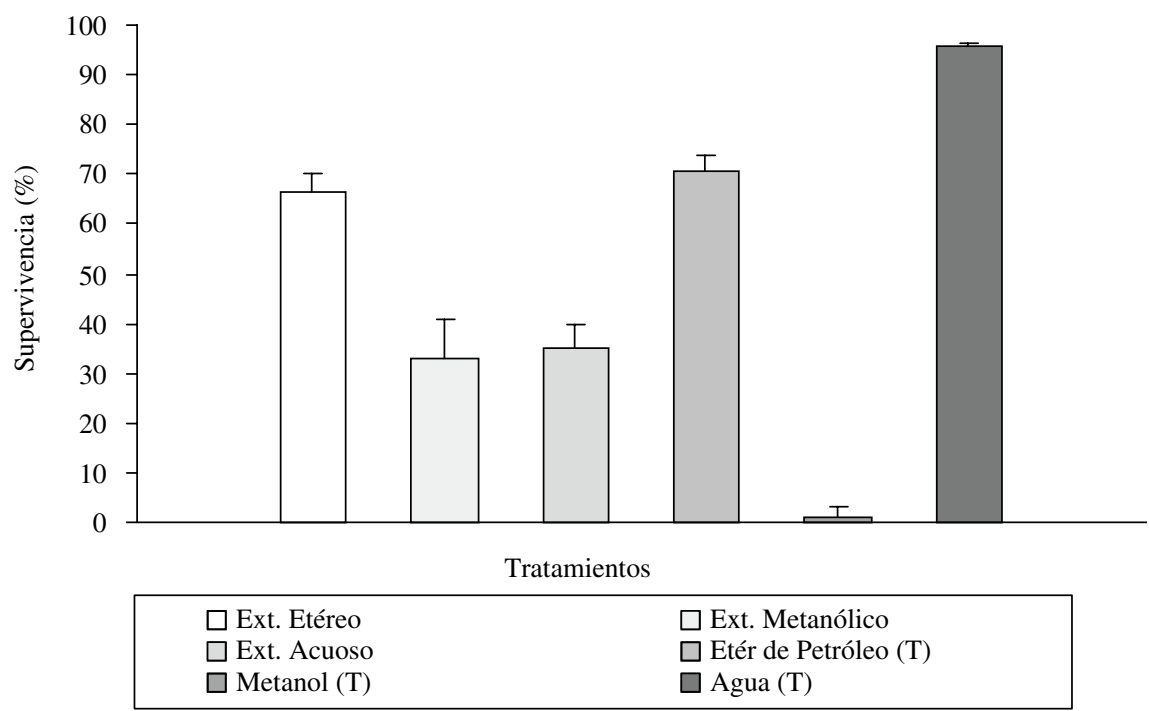

Figura 4. Supervivencia (\%) de larvas de $O$. surinamensis en los distintos tratamientos. 
relativa que resulta de la separación previa de los compuestos no polares. Los resultados obtenidos para los insectos adultos como para la descendencia de Oryzaephilus surinamensis L. demuestran un mayor efecto del conjunto de compuestos más polares de C. album contenidos en sus extractos metanólico (derivados fenólicos, flavonoides y algunas saponinas poco glicosiladas) y acuoso de hojas e inflorescencias de C. album (que contiene el mismo tipo de compuestos con un mayor grado de glicosilación). La acción conjunta de dichos metabolitos resultó más efectiva que la del extracto no polar inicial en el que se separaron hidrocarburos, alcoholes, compuestos carbonílicos y ácidos y ésteres de cadena larga, además de derivados esteroides y terpenoides.

Parecen particularmente promisorios los datos obtenidos respecto de la descendencia. Los resultados revelan sólo un 5,19\% de supervivencia de la primera generación de formas juveniles cuando se aplica el extracto acuoso de quinoa blanca, lo cual podría minimizar el impacto de las generaciones siguientes, sugiriendo su potencial utilidad en el manejo integrado de plagas.

\section{Literatura Citada}

Casadío, A. A.; Zerba, E. N.

1996 Desarrollo poblacional de Tribolium castaneum (Herbst) (Coleoptera: Tenebrionidae), en diferentes dietas y su influencia sobre la toxicidad y resistencia a malatión. Bol. San. Veg., Plagas. 22: 511-520.

Chiasson, H.; Vincent, C.; Bostanian, N.J.

2004 Insecticidal Properties of Chenopodium - Based Botanical. Journal of Economic Entomology 97 (4): 1378-1383.

Donnely, D.M.X.; Boland, G.

1994 Neoflavonoids. In: Harborne, J.B. (Ed.), The flavonoids Advance in Research since 1986. Chapman and Hall, London, pp. 261-265.

Dutcheshen, J.

2004 Method for protecting plants from bacterial diseases. Unit States Patent. 6: 743-752.

Environmental Protection Agency

2005 Saponins of Chenopodium quinoa. Biopesticides Registration Action Document PC Code 097094. Office of Pesticide Programs. Biopesticides and Pollution Prevention Division. 39 pp.

Fournier, P.

1999 Plantas Medicinales. Luxembourg. Tomo 1, 369.

Gökce, A.; Whalon, M.E.; Cam, H.; Yanar, Y.; Demirita, B.; Gören Nezhun

2007 Contact and residual toxicities of 30 plant extracts to Colorado potato beetle larvae. Archives of Phytopathology and Plant Protection 40 (6): 441-450.

Hernández, N. E.; Tereschuk, M.L.; Abdala, L.R.

2000 Antimicrobial activity of flavonoids in medicinal plants from Tafí del Valle (Tucumán, Argentina). Journal of Ethnopharmacology 73: 317-322.

Ibrahim, L.F.; Kawashty, S.A.; Baiuomy, A.R.; Shabana, M.M.;

El-Eraky, W.I.; El-Negoumy, S.I.

2007 A comparaty study of the flavonoids and some biological activities of two Chenopodium species. Chemistry of Natural Compounds, Vol. 3, 1.

Jabbar, A.; Zaman, M. A.; Iqbal, Z.; Yaseen, M.; Shamim, A. 2007 Anthelmintic activity of Chenopodium album (L.) and Caesalpinia crista (L.) against trichostrongylid nematodes of sheep. Journal of Ethnopharmacology 114: 86-91.
Jacobson, M.

1989 Botanical Pesticides (Past, Present and Future). In: Arnason, J.T; Philogene, B.J.R.; Morand, P. (Ed). Insecticides of Plant Origin. Annual of Chemistry Society. Washington: ACS, $213 \mathrm{p}$.

Lancher, W.

2000 Ecofisiologia vegetal. São Carlos: Rima. 519 p.

Leicach, S.; Della Penna, A.; Sztarker, N.; Olloquiegui, M. 2006 Efectos Alelopáticos de Residuos de Chenopodium album sobre Soja sembrada bajo la modalidad Siembra Directa Medioambiente en Iberoamérica. Visión desde la Física y la Química en los Albores del Siglo XXI. 2: 263-270. Ed. J. Gallardo Lancho, España. ISBN-13: 978-84-611-0352-2; 978-84-611-0393-5.

Marzocca, Angel

1997 Vademécum de malezas medicinales de la Argentina indígena y exótica, $1^{\text {a }}$ ed., Buenos Aires: Orientación Gráfica Editora, xix, $363 \mathrm{p}$.

Miana, G.A.; Rahman, A.U.; Iqbal, C.M.I.; Jilani, G.; Bibi, H. 1996 Pesticidas nature: Present and Future Perspectivas. In Copping, L.G. (Ed). Crop Protection Agents from Nature: Natural Products Analogues. Cambridge: RSC, pp. 241253.

Mowery, S. V.; Mullen, M. A.; Campbell, J. F.; Broce, A. B. 2002 Mechanisms underlying sawtoothed grain beetle (Oryzaephilus surinamensis [L.]) (Coleoptera: Silvanidae) infestation of consumer food packaging materials. Journal of Economic Entomology 95: 1333-1336.

Pricket, A. J.; Muggleton, J.; Llewellin, J. A.

1990 Insecticide resistance in populations of Oryzaephilus surinamensis and Cryptolestes ferrugineus from grain stores in England and Wales. Brighton Crop Protection Conference-Pests and Diseases. 3: 1189-1194.

Rodríguez, S. M.; Leicach, S. R.; Delfino, S.; Yaber Grass, M. Russo, S.; Gaglietti, D. M.

2004. Estudio exploratorio del efecto insecticida de metabolitos secundarios de Chenopodium album sobre Oryzaephilus surinamensis L. (Coleoptera: Silvanidae). Idesia. 22 (2): 61-68.

Said, M.

1969 Hamdard Pharmacopoeia of Eastern Medicine. Hamdrad Nacional Foundation, Karachi, Pakistan, 395-396. 
Saini, E. D.; Rodríguez, S. M.

2000 Insectos perjudiciales a los productos almacenados. Publicación del Instituto de Microbiología y Zoología Agrícola INTA No 7, 56 pp.

Silva Aguayo, G.A.

2001 Insecticidas vegetales. http://ipmworld.umn.edu/ cancelado/Spchapters/GsilvaSp.htm.

Sing, P.; Moore, R.F. (eds.)

1985 "Handbook of insect rearing". Vol. 1. Elsevier. Amsterdam. p. 514

Tousarkissian, $\mathrm{M}$.

1980 Plantas Medicinales de la Argentina: sus nombres Botánicos, Vulgares, Usos y Distribución Geográfica. Hemisferio Sur, Bs. As., pp. 1-39.
Wallbank, B. E.; Collins, P. J.

2003 Recent changes in resistance to grain protectants in eastern Australia, pp. 66-70. In: E. J. Wright, M. C. Webb $\&$ E. Highley (eds.). Stored grain in Australia. Proceedings of the Australian Post harvest. Technical Conference, Canberra, 25-27.

Woldu, Y.; Abegaz, B.

1990 Phythochemistry 29, 2013.

Wollenweber, E.

1994 Flavones and Flavonols. In: Harborne, J.B. (ed.), The flavonoids, Advance in Research since 1986, Chapman and Hall, London, p. 261. 
